\title{
JDBC Driver
}

National Cancer Institute

\section{Source}

National Cancer Institute. JDBC Driver. NCI Thesaurus. Code C45795.

An application program interface specification for connecting programs written in Java to the data in a database. It is a Java API that enables Java programs to execute SQL statements and interact with any SQL-compliant database. (netlingo.com) 